\title{
Process Evaluation of Sugar-Based Polymeric Colloidal Nanocarrier Formation
}

\author{
Thais Aragão Horoiwa ${ }^{a}$, Adriano Marim de Oliveira ${ }^{a *}$, Amanda Migotto ${ }^{a, b}$, \\ Natalia Neto Pereira Cerize ${ }^{a}$ \\ anstituto de Pesquisas Tecnológicas, Grupo de BioNanoManufatura, Av. Prof. Prof. Almeida Prado, \\ 532, CEP: 05508-901, São Paulo, SP, Brasil \\ ${ }^{b}$ Instituto de Ciências Biomédicas, Universidade de São Paulo, Av. Prof. Prof. Lineu Prestes, 2415, \\ CEP: 05508-900, São Paulo, SP, Brasil
}

Received: January 30, 2018; Revised: November 30, 2018; Accepted: December 13, 2018

\begin{abstract}
Several studies have demonstrated that sugar-based nanocarriers are recommended to develop drug delivery systems since they are biocompatible and biodegradable and might improve the therapeutic index of currently used drugs by reducing their toxicity and enhancing their bioavailability. Therewith, this work studied the processing conditions to formation of a sugar-based nanocarrier denominated polymeric colloidal nanocarriers (PCN). It reports the impact of emulsifiers types and concentration, salt concentration and copolymers addition on physical stability and particle size distribution of a maltodextin-based PCN. In order to define the most stable formulation to produce the maltodextrin nanocarriers, $13 \mathrm{PCN}$ formulations were evaluated in respect to kinetic stability, particle size distribution and morphology. The results demonstrated that to have a stable sugar-based PCN in silicone for at least 3 days, it is necessary to work with Poloxamer 188 as a copolymer, SF 1540 surfactant in a concentration proportional to the solid content and a salt concentration of $0.4 \%$.
\end{abstract}

Keywords: polymeric colloidal nanocarrier, block copolymer, silicone surfactants, maltodextrin.

\section{Introduction}

Nanocarriers are structures which sizes range within $1-1000 \mathrm{~nm}$ and can be arranged as liposomes, micelles, nanotubes, nanocrystals, quantum dots, dendrimers or polymeric/inorganic nanoparticles ${ }^{1,2}$. They have been developed as drug delivery devices due to their ability to improve the therapeutic index of currently used drugs by reducing their toxicity and enhancing their bioavailability ${ }^{3-5}$.

Nanocarriers introduce alternatives for inappropriate pharmacokinetic and biochemical profile drug therapies such as hydrophilic drugs which present limited interaction with lipophilic skin-layer structures, compromising their penetration through topical administration route ${ }^{6-8}$. Thus, nanocarriers may be applied to encapsulate those drugs, improving their solubility and affinity with lipophilic skinlayers by modifying their surface and changing their molecules hydrophilic-lipophilic balance (HLB) ${ }^{9,10}$.

Several materials such as lipids, proteins, polymers, metals and sugars have been used for nanocarriers development ${ }^{2}$. However, sugars have particular properties desired for drug delivery, such as stealth characteristics, bio adhesive property, biostability and solubility ${ }^{4,10}$. Furthermore, carbohydrates are biocompatible, biodegradable and non-immunogenic since they are essential components for human body metabolism ${ }^{3,10}$.

For these reasons, sugar-based nanocarriers may assist in the development of a safe, effective and stable formulation and

*e-mail: amarim@ipt.br several studies have reported their application (e.g., chitosan for pulmonary delivery ${ }^{3,11}$ or conjunctivitis treatment ${ }^{10}$, starch for topical delivery in skin cancer ${ }^{12}$, lactose for hepatic cancer treatment ${ }^{13}$ or hyaluronan for gene delivery).

In a search for a safe and low toxicity alternative to sitespecific release and topical application of hydrophilic molecules, a novel polymeric colloidal nanocarrier (PCN) based on sugar particles dispersed in silicone was proposed ${ }^{9,14,15}$. This PCN is a nanodispersion constituted by a polymeric-colloidal core containing carbohydrates and silicone emulsifiers and an external phase of silicone processed according to a previously patented methodology described in "Colloidal Nanoscale Carriers for Active Hydrophilic Substances and Method for Producing Same" (2013) ${ }^{9}$. Despite the use of carbohydrate as polymeric matrice, the silicone is an additional innovative approach in these novel nanocarriers because its molecular structure facilitate the permeation through the lipid barrier and, consequently, carries dispersed particles into deep cells improving hydrophilic drug delivery through a lipophilic route such as the topical ${ }^{12,16}$

The number of researches on these novel PCN have been rising because it has potential to introduce new topical medicines with sustained release and target delivery for hydrophilic molecules ${ }^{14,17,18}$. So, this work has been devoting attention to describe the best conditions to obtain these colloidal nanocarriers based on maltodextrin as a sugar-core polymer with acceptable physical stability, morphology and particle distribution size. 


\section{Materials}

Maltodextrin was purchased from Givaudan. Dimethicone with a viscosity of $100 \mathrm{cSt}$ was obtained from Daltomare (São Paulo, Brazil). Silicone Surfactants - Cyclopentasiloxane (and) PEG/PPG-20/15 dimethicone (SF1540 ${ }^{\circledR}$ ); PEG/PPG-20/15 Dimethicone (and) Diisopropyl Adipate (Silform60A ${ }^{\circledR}$ ) were purchased from Momentive (São Paulo, Brazil). Poloxamer 188 was purchased from Sigma-Aldrich (Germany). Analytical grade sodium chloride was used.

\section{Methods}

\subsection{Process production of maltodextrin polymeric colloidal nanocarriers (PCN)}

The PCNs were prepared as shown in Figure 1 through the methodology described in "Colloidal Nanoscale Carriers for Active Hydrophilic Substances and Method for Producing Same" $(2013)^{9}$ where a nanoemulsification followed by water extraction from the internal phase of the preformed nanoemulsion is performed.

The process of PCN preparation involves three steps as depicted in Figure 1. The first step comprises the preemulsification of aqueous solution containing maltodextrin into a silicone phase by mechanical homogenization for 5 min at 1,000 rpm (Mechanical Homogenizer, IKA model RW 20 digital). The internal phase of the nanoemulsion was prepared by dissolving polymers (maltodextrin and Poloxamer 188) into an aqueous solution containing sodium chloride $(\mathrm{NaCl})$. Silicone (dimethicone) with surfactant SF1540 or Silform 60A were used as the external phase.

The second step includes nanoemulsification by six cycles of a high-shear homogenization process in a high pressure homogenizer (Homogenizer Invensys APV, Model APV-200) operating at 1,000 bar.

During the third step, the obtained nanoemulsion was transferred to a distillation evaporator system and water of the internal phase is partially extracted by increasing the temperature up to $50{ }^{\circ} \mathrm{C}$ under vacuum and continuous stirring, inducing the maltodextrin PCN formation.

Following this production protocol, 13 experiments with different formulation conditions showed in Table 1 have been processed. The system was monitored by removing aliquots of emulsion at predetermined time intervals to define which condition could result in the best stability maltodextrin nanoparticulated system dispersed in silicone.

\subsection{Particle Size, Kinetics Stability and Morphology Characterizations}

Particle Size. The average particle size $\left(\mathrm{Z}_{\text {average }}\right)$ and polydispersity index (PI) were measured by the photon correlation spectroscopy (PCS, NanoSizer, Malvern Instruments Corp, U.K.) at $25^{\circ} \mathrm{C}$ in polystyrene cuvettes. The measurements were obtained using a He-Ne laser of $633 \mathrm{~nm}$. Samples were dispersed in a mixture of $2 \% \mathrm{w} / \mathrm{w}$ Silform $60 \mathrm{~A}^{\circledR}$ silicone in SF $1202^{\circledR}$ silicone, at a proper dilution. The dispersive solution parameter refraction index (1.396 at $\left.25^{\circ} \mathrm{C}\right)$ and the viscosity ( $\left.4.1 \mathrm{cPs}\right)$ were used to calculate the particle size. The measurements were performed in triplicate and the averaged results concerning particle size and PI are reported herein.

Kinetics stability. The kinetics stability of the new nanocarrier was evaluated using a Turbiscan ${ }^{\circledR}$ Lab (Formulaction, model MA 2000, France). The PCNs were transferred to a cylindrical glass cell and analyzed by a light beam emitted in near infrared $(880 \mathrm{~nm})$ wavelength which vertically scanned the sample cell. Two synchronous optical

\section{External phase \\ Silicone + Emulsifier \\ Internal phase \\ Water + Polymers}
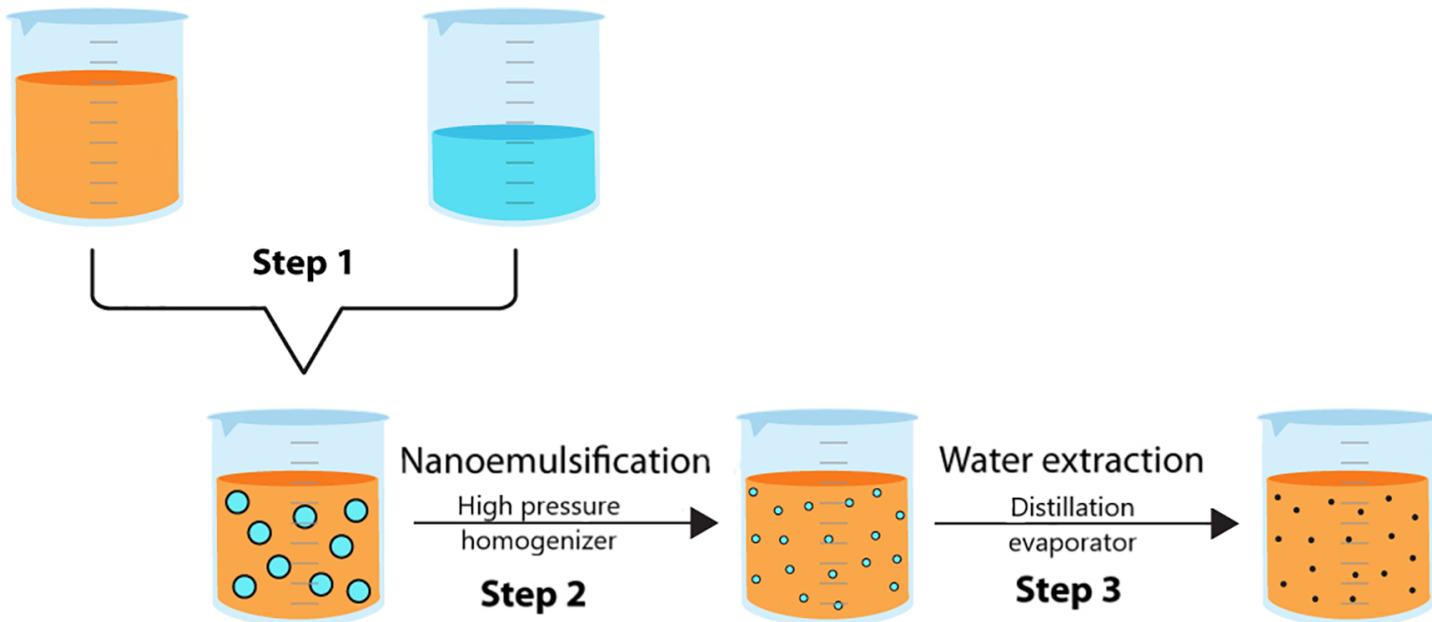

Figure 1. Preparation process of Polymeric Colloidal Nanocarriers (adapted from N.N.P. Cerize, $2012^{2}$. 
Table 1. Processed Formulation of Maltodextrin PCN.

\begin{tabular}{|c|c|c|c|c|c|c|c|}
\hline \multirow{3}{*}{ Batch } & \multicolumn{4}{|c|}{ Internal phase } & \multicolumn{3}{|c|}{ External phase } \\
\hline & \multirow{2}{*}{$\begin{array}{c}\text { Maltodextrin } \\
\% \mathrm{w} / \mathrm{w}\end{array}$} & \multirow{2}{*}{$\begin{array}{c}\begin{array}{c}\text { Poloxamer } \\
188\end{array} \\
\% \mathrm{w} / \mathrm{w}\end{array}$} & \multirow{2}{*}{$\begin{array}{c}\text { Salt } \\
\% \text { w/w }\end{array}$} & \multirow{2}{*}{$\begin{array}{l}\text { Water } \\
\% \text { w/w }\end{array}$} & \multicolumn{2}{|c|}{ Emulsifier } & \multirow{2}{*}{$\begin{array}{l}\text { Silicone } \\
\% \text { w/w qs }\end{array}$} \\
\hline & & & & & Type & $\% \mathrm{w} / \mathrm{w}$ & \\
\hline 1 & 5 & - & 0.3 & 25 & SF1540 & 5 & 100 \\
\hline 2 & 5 & - & 0.3 & 24 & SILFORM 60-A & 5 & 100 \\
\hline 3 & 9 & - & 0.9 & 20 & SF 1540 & 5 & 100 \\
\hline 4 & 5 & - & 0.9 & 24 & SF1540 & 5 & 100 \\
\hline 5 & 5 & - & 0.0 & 25 & SF 1540 & 5 & 100 \\
\hline 6 & 4 & - & 0.0 & 26 & SF 1540 & 5 & 100 \\
\hline 7 & 5 & 4 & 0.4 & 21 & SF1540 & 5 & 100 \\
\hline 8 & 5 & 4 & 0.4 & 21 & SILFORM 60-A & 10 & 100 \\
\hline 9 & 5 & 4 & 0.4 & 21 & SF 1540 & 10 & 100 \\
\hline 10 & 5 & 4 & 0.4 & 21 & SF1540 & 10 & 100 \\
\hline 11 & 5 & 0.5 & 0.4 & 24 & SF 1540 & 10 & 100 \\
\hline 12 & 5 & - & 0.4 & 25 & SF 1540 & 10 & 100 \\
\hline 13 & 5 & 4 & 0.4 & 21 & SF 1540 & 5 & 100 \\
\hline
\end{tabular}

sensors received respectively the light transmitted through the sample and the light backscattered by the sample. The backscattering (BS) was directly dependent on the particle mean diameter. The sample in the cell was scanned every hour for three days at $25^{\circ} \mathrm{C}$ and the $\mathrm{BS}$ variation obtained was used for obtaining the computation Turbiscan Stability Index (TSI). The TSI is calculated by the total sum of all the BS variations in the sample to provide, as a result, a unique number reflecting the destabilization of a given sample and it is used as a measure of the formulations stability. TSI value is positively related to sample destabilization.

Morphology. The morphology was evaluated by using the Field Emission Gun Scanning Electron Microscopy technique (microscope FEG-SEM, Model Quanta 3D, FEI). The sample preparation protocol consisted of a vacuumassisted membrane filtration using $0.22 \mu \mathrm{m}$ cellulose esther membranes followed by rinsing with cyclohexane to remove silicone excess still present in the sample. The membrane with the harvested particles was dried on a desiccator under vacuum during at least $24 \mathrm{~h}$. Afterwards, the membrane with particles was sputter-coated to deposit a thin layer of Au-Pd.

The operating procedure of the microscope considered the use of the high-vacuum mode and an accelerating voltage of $20 \mathrm{kV}$.

\section{Results and Discussion}

\subsection{Production process evaluation of maltodextrin PCN}

The evaluation of 13 prepared batches of maltodextrin PCN showed that, following the previous described protocol, it is possible to obtain a white opaque and fluid dispersion
(Figure 2) of regular shape maltodextrin nanoparticles in silicone with a mean diameter ranging from $233 \mathrm{~nm}$ to $460 \mathrm{~nm}$ and polydispersity index ranging from 0.07 to 0.35 as shown in Table 2.

Based on characterization results of the 13 processed experiments different formulation conditions have been evaluated and compared considering their kinetic stability defined by their TSI results which are shown in Table 2 . The variants were: two kinds of surfactant (SF1540 and Silfom60-A), two different concentrations of these surfactants $(5 \% \mathrm{w} / \mathrm{w}$ or $10 \% \mathrm{w} / \mathrm{w})$, four different concentrations of sodium chloride $(0.0 \%, 0.3 \%, 0.4 \%$ or $0.9 \%$ in mass of total formulation) and the addition or not of copolymer Poloxamer 188 in the internal phase. Therewith, following studies were structured to guide the selection of the most stable formulation to nanocarriers defined by the lowest TSI.

\section{- Emulsifiers types}

Among different surfactants available on the market indicated for water-in-silicone emulsion stabilization, we selected two of them to perform the study: Silform60A and SF1540. The selected surfactants are a polyether-functionalized siloxane composed of organic/inorganic hybrid polymeric molecules with silicon atoms alternated with oxygen and metil groups ${ }^{16,19,20}$. The specific weight ratio of polyether to silicone in these commercial surfactants creates an amphiphilic structure that abilities them interact either to lipophilic external phase and hydrophilic polymeric internal phase on the proposed nanocarrier stabilizing its sugar-based nanoparticles dispersion on silicone $e^{9,19,20}$.

Batch 8 was produced using Silform 60A and Batch 9 was produced using SF1540. As can be observed in Figure 3, the TSI value (15.9) of Batch 9, for which $10 \%$ $(\mathrm{m} / \mathrm{m})$ of SF 1540 has been used, was lower than the TSI 


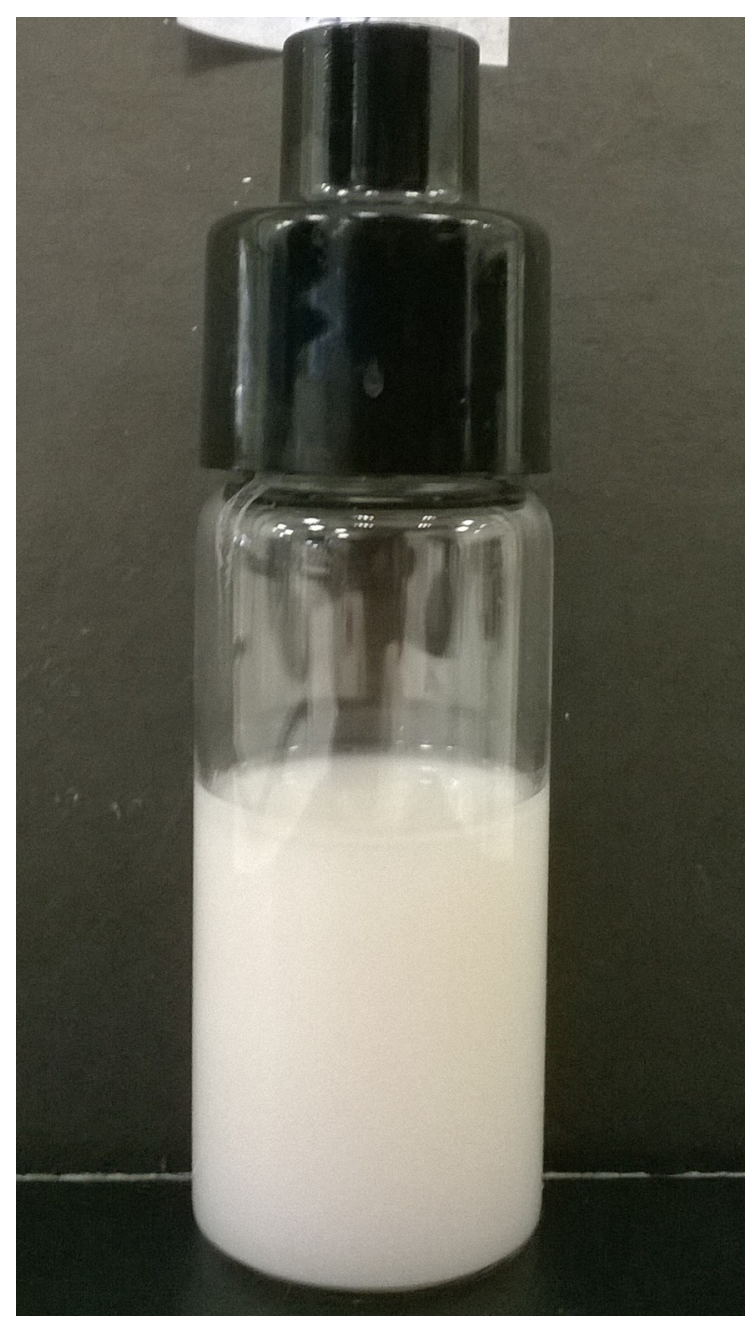

Figure 2. Maltodextrin nanoparticle dispersion in silicone.

Table 2. Characterization Results of Maltodextrin PCN.

\begin{tabular}{lllc}
\hline \multirow{2}{*}{ Batch } & \multicolumn{2}{c}{ Particle Size } & $\begin{array}{c}\text { Physical Stability } \\
\text { (3 days) }\end{array}$ \\
\cline { 2 - 4 } & nm & PI* & TSI \\
\hline 1 & 345 & 0.22 & NE \\
\cline { 2 - 4 } 2 & & Coagulated \\
3 & \multicolumn{3}{r}{ Coagulated } \\
4 & \multicolumn{3}{c}{ Coagulated } \\
5 & 269 & 0.21 & NE \\
6 & 310 & 0.35 & NE \\
7 & 406 & 0.21 & NE \\
8 & 269 & 0.21 & 27.1 \\
9 & 279 & 0.19 & 15.9 \\
10 & 281 & 0.07 & 11.7 \\
11 & 233 & 0.08 & $18.4 * *$ \\
12 & 263 & 0.15 & 20.1 \\
13 & 393 & 0.14 & 16.6 \\
\hline
\end{tabular}

*PI = Polydispersity Index;

$* * 2$ days evaluation;

$\mathrm{NE}$ - non-evaluated value (27.1) of Batch 8 , for which $10 \%(\mathrm{~m} / \mathrm{m})$ of Silform $60 \mathrm{~A}$ has been used. In addition, results for Batch 2 shows that $5 \%$ of Silform- $60 \mathrm{~A}$ are not able to stabilize properly the polymer core in silicone as coagulation of particles was detected (Figure 4).

The instability of Batch 2 can be explained by the presence of diisopropyladipate in the Silform 60A commercial reagent used for dispersing the polymeric chains of the surfactant. The optimal interaction between emulsifier chains, internal and external phase of the system is hindered by diisopropyladipate because this solvent has no great solubility in the silicone external phase.

The SF1540, in turn, has no problem with phase incompatibility because the solvent used to disperse this surfactant is cyclopentasiloxane. This solvent has a chemical structure similar to silicone used in the external phase of the nanocarrier dispersion which allows better solubilization of the emulsifier in the system and, consequently, provides more freedom for the surfactant allowing the interaction between system phases as represented in Figure 5C. Therefore, SF1540 is considered the most indicated surfactant for producing maltodextrin nanocarrier systems dispersed in silicone.

- Surfactant Concentration

In order to evaluate the influence of emulsifier concentration in the stabilization of nanocarriers, samples were tested with 5 and $10 \%$ of SF 1540 emulsifier. Figure 6 shows the kinetic stability of Batch 13, with $5 \%$ SF 1540 , and Batch 10 , with $10 \%$ of the same emulsifier. It can be observed that the highest concentration of SF1540 (10\%) was more efficient in stabilizing nanocarriers suspended in silicone showing lower TSI variation $(\mathrm{TSI}=11.7)$ than the sample with $5 \%$ SF 1540 (TSI= 16.6).

Similarly, Mehta et al. (2009) demonstrated that the droplets size was decreased and the uniformity of silicone emulsions was improved with the increase in silicone-based emulsifier concentration, which enhanced of emulsions stability $^{21}$. Although, Fengyan et al. (2011) found that the size distribution became narrow with the increase in concentration of emulsifier for silicone emulsions prepared by Span-60 emulsifier. It leads them proposed that an excessive amount of emulsifier can be increase the viscosity of silicone oil emulsion with high solid content causing a broad size distribution $^{21,22}$.

These findings favored the optimum amount of emulsifier to prepare stable silicone oil emulsions is requested and to maintain a stable maltodextrin nanoparticles dispersion in silicone the optimal emulsifier concentration is proportional to the solid content. Therefore, as our goal is to obtain a solid content of about $10 \%$, we decided to use $10 \%$ of SF 1540 to produce more stable maltodextrin PCN.

- Sodium Chloride Concentration

The presence of electrolytes increases the ionic interaction strength of the polymer chain with the hydrophilic phase reaching an optimal orientation of the molecular structure 


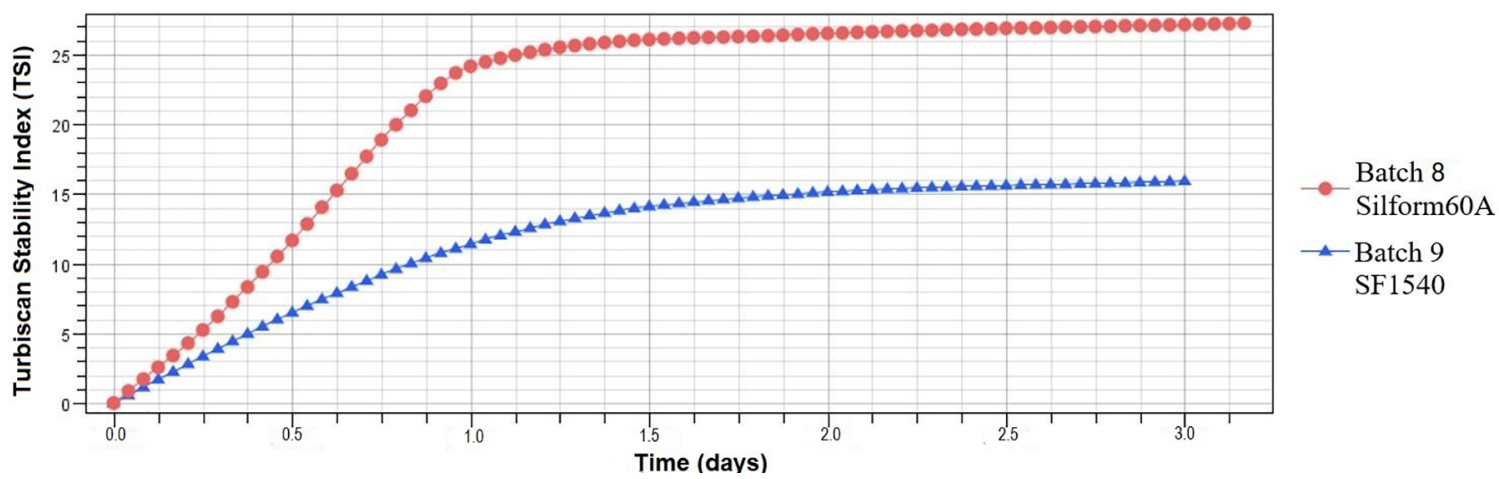

Figure 3. Kinetic-stability comparison of Batch 8 and Batch 9.

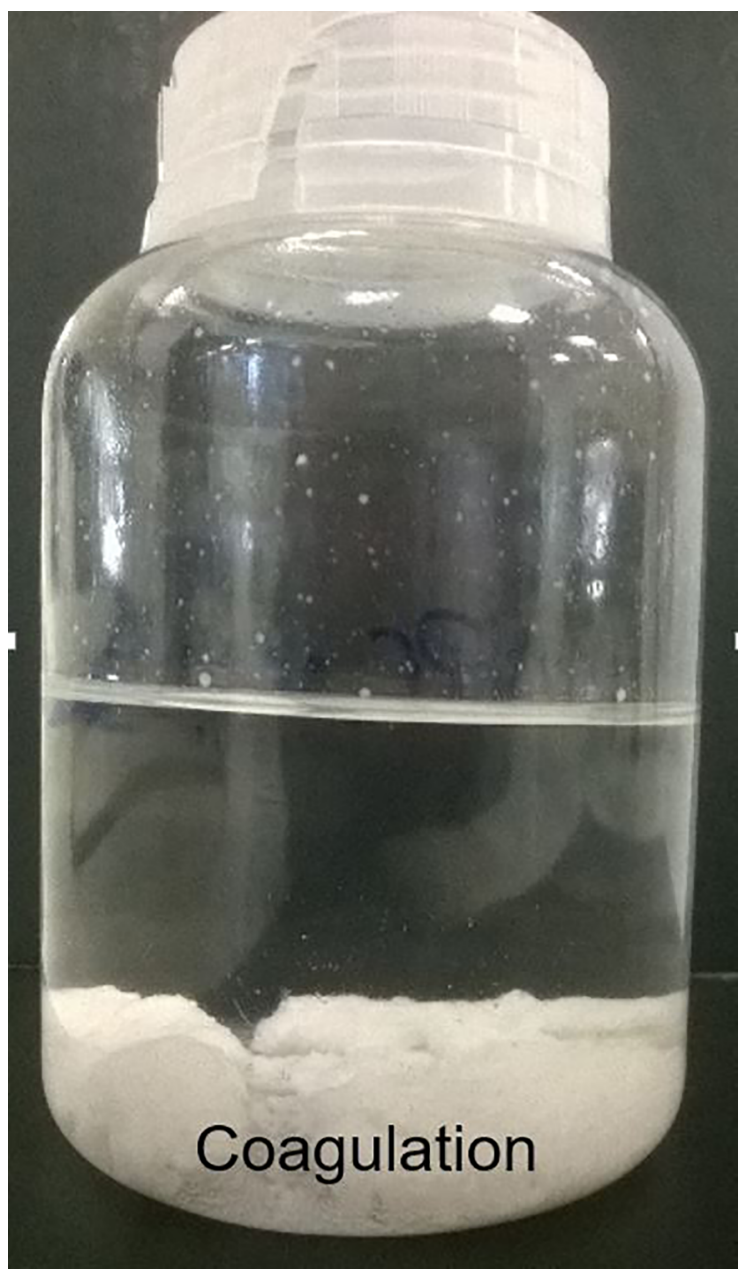

Figure 4. Polymer coagulation of Batch 2.

of the emulsifier/water interface silicone as represented in Figure $5 \mathrm{C}^{16}$. Therefore, different concentrations of sodium chloride in the aqueous phase were designed to evaluate the influence of the concentration of the electrolyte in stabilizing nanocarriers. Observing the physical aspects of formulations processed in Batch 4 and Batch 6, we were able to conclude that to stabilize the maltodextrin particles suspended in silicone nanocarrier, the concentration of $\mathrm{NaCl}$ should be equal or higher than $0.3 \%$ and lower than $0.9 \%$.

This conclusion was reached because an agglomeration has been observed in the formulation with $0.9 \% \mathrm{NaCl}$ (Batch 4) shown in Figure 7. In contrast, the formulation without $\mathrm{NaCl}$ (Batch 6) has not shown any agglomeration but presented phase separation as shown in Figure 8.

These results indicate that there is an ideal sodium chloride concentration for siloxane emulsifier to achieve the best molecular orientation to promote the optimal interaction between the polymer core and the silicone external phase. This can be attributed to the mechanism of emulsion stabilization associated with silicone emulsifiers used in the PCN production. The emulsifiers used consist of siloxane functionalized polyether chains. These chains may be positioned at the liquid interface across multiple segments as represented in Figure 5C and the adsorption energy is the sum of the interaction of all segments ${ }^{16}$. The stronger the interactions between polymer chains of the emulsifier dispersant phase, the more effective is the emulsion stabilization process ${ }^{23}$.

Binks et al. (1999) have also demonstrated that there is an optimal range of concentration of sodium chloride which keeps the ability of emulsifiers to stabilized mixtures containing silicone (polydimethylsiloxane - PDMS) and water using anionic surfactant AOT. They found that a water-insilicone emulsions are forming around salt concentrations which the interfacial tension is minimum. This finding was rationalized in terms of changes in the spontaneous curvature of the monolayer coating the microemulsion drops, driven by variations in the effective geometry in situ of the emulsifier at the silicone/water interface ${ }^{24}$.

A correlation between Binks' findings and the events observed in the presented study can be established and explains that the best salt concentration to achieve a stable maltodextrin PCN system was $0.4 \% \mathrm{NaCl}(\mathrm{w} / \mathrm{w})$ because interfacial tension might be minimum in that condition and favorable molecular orientation of the SF1540 emulsifier is reached. It keeps the hydrophilic internal phase interfacing with the silicone external phase, avoiding sedimentation or coalescence of the PCN polymeric core. 


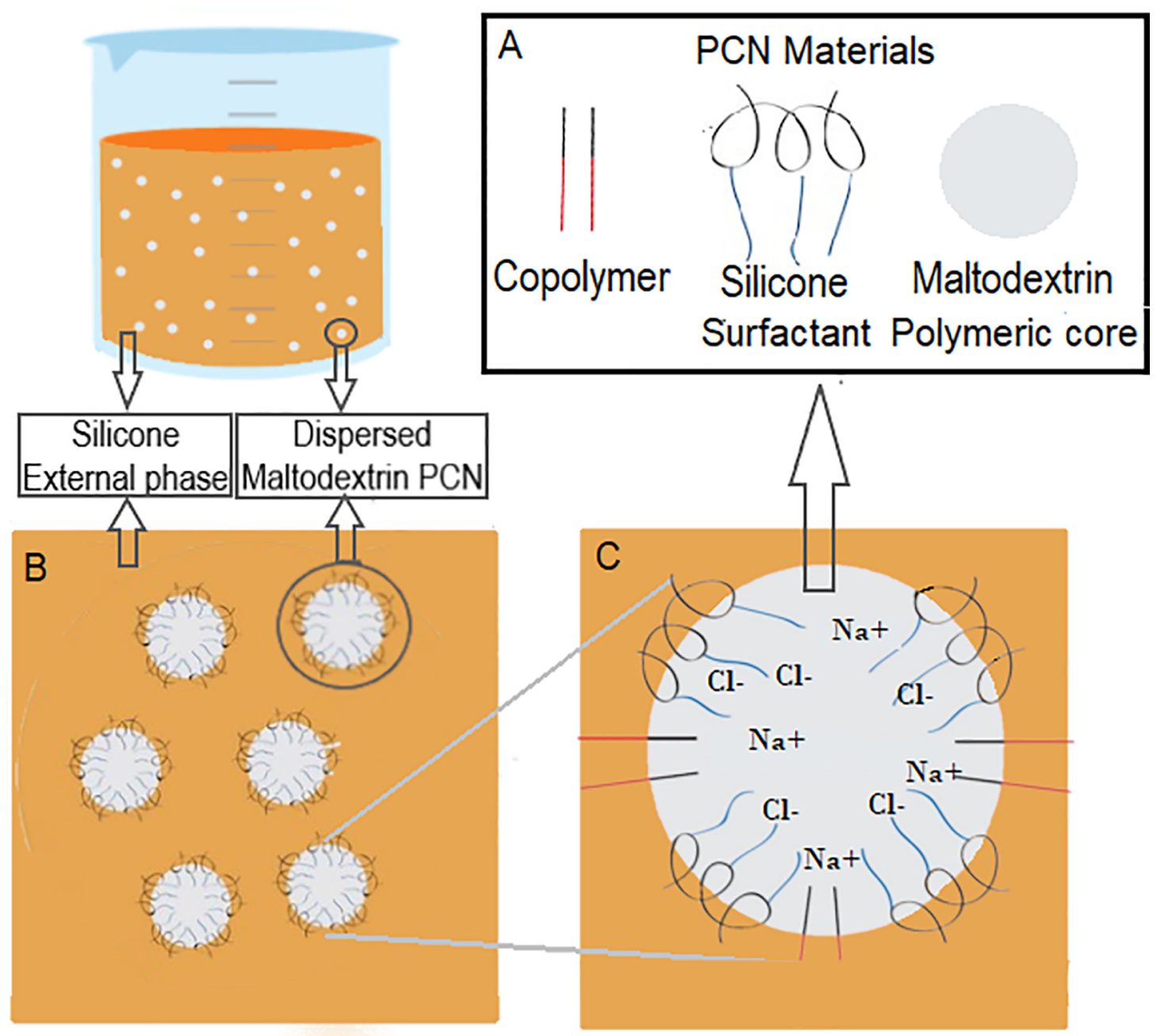

Figure 5. Schematic representation of maltodextrin PCN dispersion in silicone (A) maltodextrin PCN materials composition. (B)Amplification of maltodextrin PCN dispersion in silicone. (C) Representation of maltodextrin PCN materials disposition in silicone dispersion.

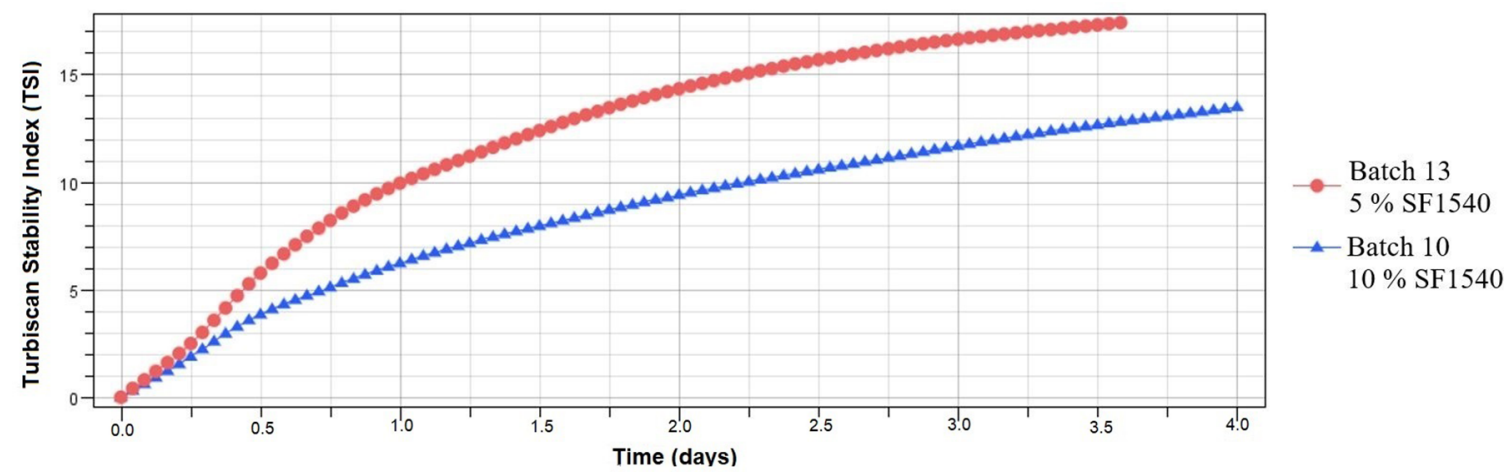

Figure 6. Kinetic stability comparison of Batch 13 and Batch 10. 


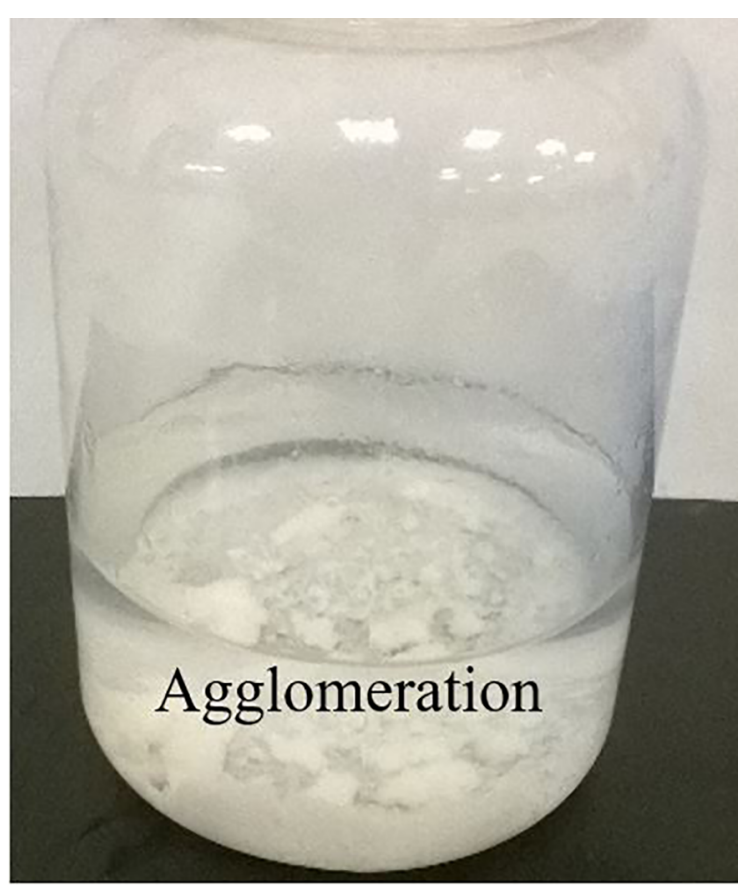

Figure 7. Agglomeration in Batch 4.

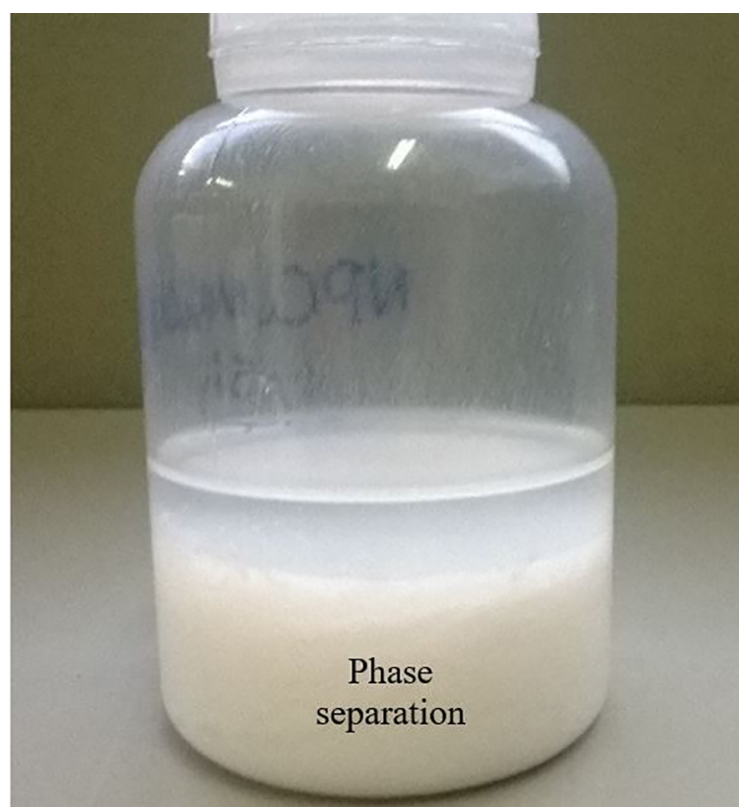

Figure 8. Phase separation in Batch 6.

\section{- Copolymer addition}

The addition of Poloxamer improved kinetic stability of nanocarriers when compared with the kinetic stability of formulations processed in Batch 10 and Batch 12, as shown in Figure 9.
In this Figure, it can be observed that the sample with $4 \%$ Poloxamer (Batch10 - red curve) has lower TSI after $24 \mathrm{~h}$, being 11.7 on the third day of analyses. Although, even with lower TSI variation during the first day of analyses (TSI 1 day $=5.8$ ), the sample without Poloxamer (Batch 12 - blue curve) shows an exponential growth trend after two days, presenting a TSI value equal to 20.1 on the third day which is higher than the sample containing $4 \%$ Poloxamer. These results indicate that the use $4 \%$ Poloxamer as a copolymer in the structure of maltodextrin nanocarriers improves stability of the dispersion of maltodextrin nanoparticles.

This result may be a consequence of the Poloxamer being a block copolymer with physicochemical characteristics of non-ionic emulsifier. These features are due to its bifunctional monomers in the molecular structure (Figure 10) consisting of two polyethylene glycol (x) and polypropylene (y), which have different solubility, providing the molecule an amphiphilic profile and ability to perform an emulsifier function ${ }^{25,26}$.

Due to this bifunctional characteristic, it can be suggested that the Poloxamer interact with maltodextrin chains through its hydroxyl group terminal portion forming a grafted copolymer. That hypothesis arises considering that Poloxamer can be coupled with other polymers such as poly(acrylic acid) (PAA) via $\mathrm{C}$ - $\mathrm{C}$ bonding by simple radical polymerization as Bromberg previously described ${ }^{27,28}$. These grafted polymers have the ability to form temperature dependent micellar aggregates and, after a further temperature increase, gels due to micelles aggregation or packing ${ }^{26,27}$.

Similar mechanism of polymerization between Poloxamer and maltodextrin is proposed for PCN formation and the same thermosensitive gelling polymer properties plays a role in the increase of PCN kinetic stability because polymers gel phase promotes a steric hindrance stabilization mechanism to maltodextrin nanocarrier.

Considering this proposed mechanism and the stability evaluations of samples with and without Poloxamer, the formulations containing concentrations of $4 \%$ of emulsifier have been chosen to obtain a system with improved kinetic stability.

\subsection{Maltodextrin colloidal nanocarriers: optimized formulations}

The kinetic stability studies showed that the formulation containing $5 \%$ of maltodextrin, $4 \%$ of Poloxamer, $0.4 \%$ $\mathrm{NaCl}$ and $10 \%$ of SF 1540 is the best formulation in order to obtain the highest kinetic stability among tested conditions. Therewith, two batches (Batch 9 and Batch 10) have been processed under these conditions to reproducibility evaluation 


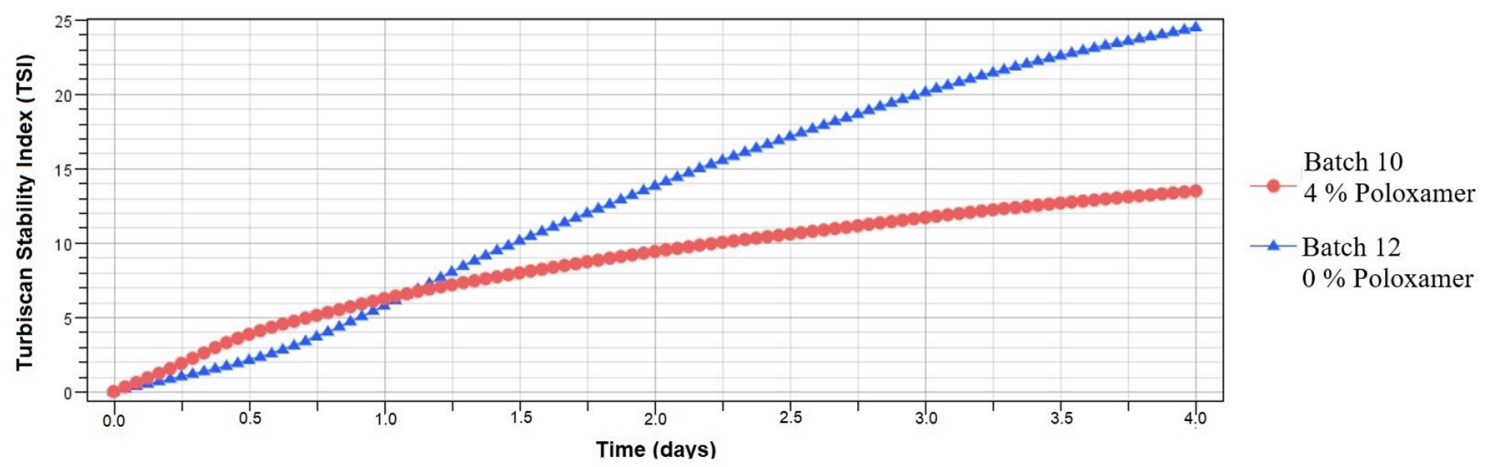

Figure 9. Kinetic stability comparison of Batch 10 and Batch 12 .

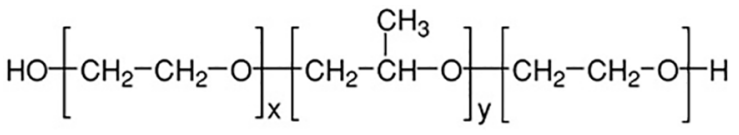

Figure 10. Poloxamer molecular structure.

as well as it was analyzed in terms of particle size and morphology showing results described below.

\section{- $\quad$ Particle Size}

Size distribution shown in Figures 11 and 12 demonstrated that maltodextrin PCN obtained by the described protocol are structures organized in nanometric scale $($ Batch $9=279 \mathrm{~nm}$; Batch $10=281 \mathrm{~nm})$ with low polydispersity index (Batch $9=0.19$; Batch $10=0.07$ ).

- Morphology of maltodextrin-PCN

The photomicrographs of maltodextrin nanocarriers shown in Figure 13 display spherical nanostructures organized in irregular agglomeration. The irregular agglomeration is due to the colloidal nature of sugar-based nanocarrier which aggregate during the process of silicone removal by cyclohexane and particles deposition in membrane for microscopy preparation.

Nevertheless, when they are analyzed individually the particles have a smooth and regular surface with relatively small size distribution, confirming the nanometric scale.

Similar results were observed in a previous patented work (US20130197100 A1) ${ }^{9}$ which first reported the PCN production using carbohydrates such as starch, chitosan and polyvinylpyrrolidone as a polymeric core associated to a silicone-based external phase. That patent has examples of PCN with particles size ranged between 168 to $505 \mathrm{~nm}$ and low polydispersity index, produced using $2 \% \mathrm{w} / \mathrm{w}$ of SF1540 as a surfactant, $0,4 \%$ of $\mathrm{NaCl}(\mathrm{w} / \mathrm{w})$ and no copolymer addition.

The present work evidences that it is possible introduces other sugar polymers as a core for $\mathrm{PCN}$ formation with similar results of the patented system and brings more information about process conditions to obtain the patented PCN using a novel carbohydrate (maltodextrin) which was not previously tested. Moreover, this work contributed to the elucidation of PCN formation mechanism as well as included more suitable options to improve the system stabilization such as copolymer addition.

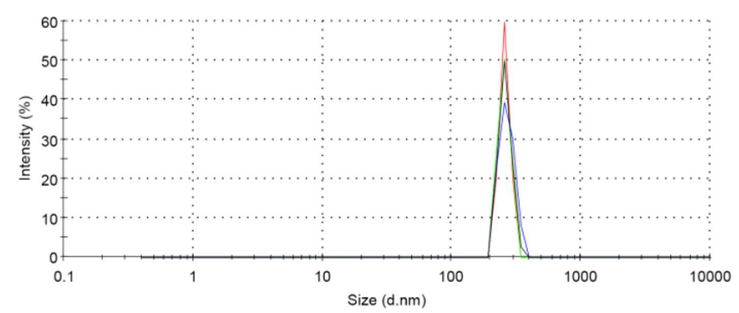

Figure 11. Batch 9 size distribution $x$ intensity.

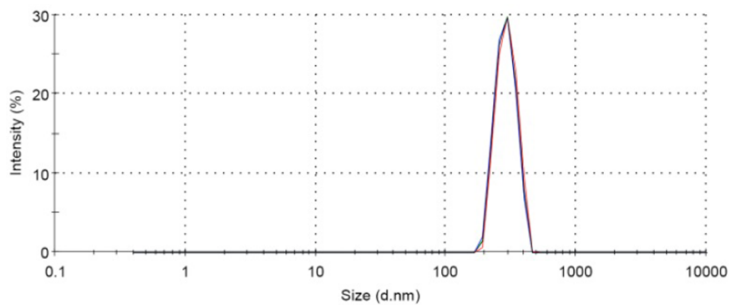

Figure 12. Batch 10 size distribution $\mathrm{x}$ intensity

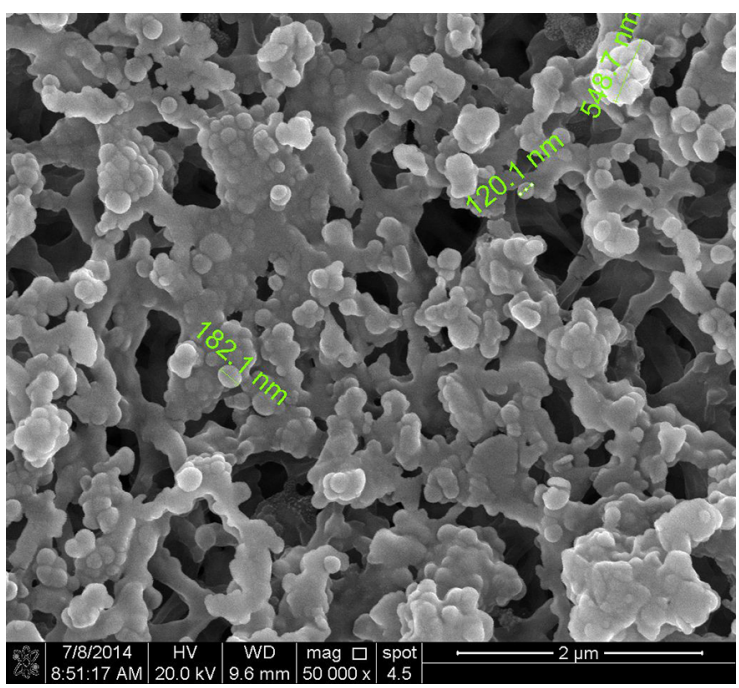

Figure 13. Morphology of maltodextrin PCN (Batch 10). 


\section{Conclusions}

This work showed that $0.4 \% \mathrm{NaCl}$ (w:w) is the best condition to keep a stable interface between the hydrophilic internal phase and the silicone-based external phase for the maltodextrin PCN formation. Moreover, SF1540 is considered the most indicated surfactant for producing stable maltodextrin nanocarrier systems in silicone, using surfactant concentrations proportional to the solid content.

Also, the Poloxamer addition proved to be an alternative as a matrice modifier of maltodextrin particles because it demonstrated a better stability in silicone suspensions than the system without this surfactant. Moreover, the maltodextrin PCN with Poloxamer could be one more alternative for the development of controlled drug delivery systems due to the solubility improvement offered by this copolymer, showing the potential to obtain a formulation for topical or oral applications.

\section{Acknowledge}

The authors would like to thank Dr. Carolina Rediguieri for her contribution on revision of the manuscript.

\section{References}

1. Bhatia S. Nanoparticles Types, Classification, Characterization, Fabrication Methods and Drug Delivery Applications. In: Bhatia S. Natural Polymer Drug Delivery Systems. Cham: Springer; 2016. p. 33-39.

2. Bobo D, Robinson KJ, Islam J, Thurecht KJ, Corrie SR. Nanoparticle-Based Medicines: A Review of FDA-Approved Materials and Clinical Trials to Date. Pharmaceutical Research. 2016;33(10):2373-2387.

3. Ngwuluka NC. Responsive polysaccharides and polysaccharidesbased nanoparticles for drug delivery. In: Makhlouf ASH, AbuThabit NY, eds. Stimuli Responsive Polymeric Nanocarriers for Drug Delivery Applications. Volume 1. Sawston: Woodhead Publishing; 2018. p. 531-354.

4. Jain K, Kesharwani P, Gupta U, Jain NK. A review of glycosylated carriers for drug delivery. Biomaterials. 2012;33(16):4166-4186.

5. Feitosa VA, Balogh TS, Guimarães KL, Zanin MHA, Oliveira AM, Cerize NNP. Skin permeation of hydrophobic drugs loaded into polymeric nanoparticles. Advanced Materials - TechConnect Briefs. 2016:79-81.

6. Shim J, Seok Kang H, Park WS, Han SH, Kim J, Chang IS. Transdermal delivery of mixnoxidil with block copolymer nanoparticles. Journal of Controlled Release. 2004;97(3):477-484.

7. Pardeike J, Hommoss A, Müller RH. Lipid nanoparticles (SLN, NLC) in cosmetic and pharmaceutical dermal products. International Journal of Pharmaceutics. 2009;366(1-2):170184.
8. Cevc G. Lipid vesicles and other colloids as drug carriers on the skin. Advanced Drug Delivery Reviews. 2004;56(5):675-711.

9. Cerize NNP, de Oliveira AM, Ré MI, Tedesco AC, inventors Universidade de São Paulo, Fundacao de Amparo a Pesquisa do Estado de São Paulo, Instituto de Pesquisa Tecnológicas do Estado de São Paulo S/A, assignees. Colloidal Nanoscale Carriers for Active Hydrophilic Substances and Method for Producing Same. United States patent; US 20130197100A1. 2013 Aug 1.

10. Zhang Y, Chan JW, Moretti A, Uhrich KE. Designing polymers with sugar-based advantages for bioactive delivery applications. Journal of Controlled Release. 2015;219:355-368. DOI: http:// dx.doi.org/10.1016/j.jconrel.2015.09.053

11. Dua K, Bebawy M, Awasthi R, Tekade RK, Tekade M, Gupta G, et al. Application of Chitosan and its Derivatives in Nanocarrier Based Pulmonary Drug Delivery Systems. Pharmaceutical Nanotechnology. 2017;5(4):243-249.

12. Cerize NNP. Estudo de sistemas nanocarreadores para o ácido 5-aminolevulínico com aplicação na terapia fotodinâmica. [Thesis]. Ribeirão Preto: Universidade de São Paulo; 2012.

13. Di Stefano G, Fiume L, Baglioni M, Busi C, Bolondi L, Farina $\mathrm{C}$, et al. Doxorubicin coupled to lactosaminated albumin: Effect of heterogeneity in drug load on conjugate disposition and hepatocellular carcinoma uptake in rats. European Journal of Pharmaceutical Sciences. 2008;33(2):191-198.

14. Horoiwa TA, Oliveira AM, Cerize NNP. Development of Polymeric Colloidal Nanocarrier for Topical Meglumine Antiomoniate Delivery. In: 66th Canadian Chemical Engineering Conference; 2016 Oct 16-19; Québec City, QC, Canada.

15. Cerize NNP, Horoiwa TA, Oliveira AM. Preparation and characterization of innovative polymeric colloidal nanocarriers based on maltodextrin. In: TechConnect World 2015; 2015 Jun 14-17; Washington, DC, USA

16. Somasundaran P, Mehta SC, Purohit P. Silicone emulsions Advances in Colloid and Interface Science. 2006;128-130:103109.

17. Horoiwa TA, Oliveira AM, Cerize NNP. Influence of block copolymer addition on polymeric colloidal nanocarriers stability. In: 10th International Congress of Pharmaceutical Sciences - CIFARP; 2015 Sep 5-9; Ribeirão Preto, SP, Brazil.

18. Horoiwa TA, de Oliveira AM, Cerize NNP. Perfil de liberação in-vitro do antimoniato de meglumina encapsulado por nanocarreadores poliméricos coloidais. Infarma, Ciências Farmacêuticas. 2018;30(1 Suppl):235.

19. Momentive. SF1540: Technical Data Sheet [Internet]. Volume 14402, HCD-14402; 2016 [cited 2018 Oct 29]. Available from: $<$ https://www.momentive.com/en-us/products/tds/sf1540/> Access in: 29/10/2018.

20. Momentive. SilForm *60-A Emulsifier: Technical Data Sheet [Internet]. Volume 26911; 2015. p. 54-55. Available from: $<$ http://www.latro.com.tr/upload/1485162766-t2.pdf>. Access in: $29 / 10 / 2018$. 
21. Mehta SC, Somasundaran P, Kulkarni R. Variation in emulsion stabilization behavior of hybrid silicone polymers with change in molecular structure: Phase diagram study. Journal of Colloid and Interface Science. 2009;333(2):635-640. DOI: http://dx.doi. org/10.1016/j.jcis.2009.01.028

22. Nazir H, Zhang W, Liu Y, Chen X, Wang L, Naseer MM, et al. Silicone oil emulsions: strategies to improve their stability and applications in hair care products. International Journal of Cosmetic Science. 2014;36(2):124-133.

23. Hill RM. Silicone surfactants - New developments. Current Opinion in Colloid \& Interface Science. 2002;7(5-6):255-261.

24. Binks BP, Dong J, Rebolj N. Equilibrium phase behaviour and emulsion stability in silicone oil+water+AOT mixtures. Physical Chemistry Chemical Physics. 1999;1(9):2335-2344.
25. Grallert RSM, Rangel-Yagui CO, Pasqualoto KFM, Tavares LC. Polymeric micelles and molecular modeling applied to the development of radiopharmaceuticals. Brazilian Journal of Pharmaceutical Sciences. 2012;48(1):1-16.

26. Jeong B, Kim SW, Bae YH. Thermosensitive sol-gel reversible hydrogels. Advanced Drug Delivery Reviews. 2002;54(1):3751 .

27. Bromberg L. Novel Family of Thermogelling Materials via C-C Bonding between Poly(acrylic acid) and Poly(ethylene oxide)-b-poly(propylene oxide)-b-poly(ethylene oxide). The Journal of Physical Chemistry B. 1998;102(11):1956-1963.

28. Bromberg L. Polyether-Modified Poly(acrylic acid): Synthesis and Applications. Industrial \& Engineering Chemistry Research. 1998;37(11):4267-4274. 